\title{
EDUCACIÓN EN TRABAJO SOCIAL: DESAFÍOS A SER ENFRENTADOS ${ }^{1}$
}

\author{
Dr. Víctor I. García Toro, Catedrático
}

\section{Introducción}

P iertamente la superación de la educación y la práctica en trabajo social para efectos de esta presentación, ha creado un vacío artificial difícil de lidiar en el contexto de la realidad profesional. Faherty (1997) en su trabajo sobre la necesidad de predicción en el trabajo social, cita a Macarov (1991, p. 176), quien al referirse al binomio educación y práctica en trabajo social, comentó:

La educación está usualmente rezagada, detrás de la práctica. Los cambios curriculares son hechos porque la práctica ha cambiado y raramente la práctica cambia porque haya habido cambios en la educación. Esto crea una desafortunada laguna durante la cual los trabajadores sociales usan teorías irrelevantes y fuera de moda, al igual que conceptos y actividades, hasta que la nueva situación es reconocida y la educación en trabajo social responde.

Es, por así decirlo, una relación simbiótica y necesaria para la profesión que garantiza su actualización y la constante necesidad de responder. Para Morales y Sheaford (1996) el trabajo social debe estar en la línea de frente, donde, apoyado por la educación en trabajo social, propicie el desarrollo en programas sociales que respondan a las necesidades humanas, como a las del deambulante, la pobreza, la ruptura en la familia, enfermedades mentales, incapacidades físicas

\footnotetext{
${ }^{1}$ Trabajo preparado por el Dr. Víctor I. García Toro, Director de la Escuela Graduada de Trabajo Social Beatriz Lassalle, en Reunión Cumbre de OLASW, Hotel Cerromar, Dorado, Puerto Rico, 12 de junio de 1998.
} 
y mentales, abuso de alcohol y substancias, violencia doméstica y otros problemas. Todo este contexto requeriría que el trabajo social estuviese en contacto con los cambios sociales que acontecen en la sociedad y que por su naturaleza requiere tanto de una educación como de una práctica en constante cambio, producción y adaptación, teniendo como base la flexibilidad y adaptabilidad hacia la respuesta en programas y servicios.

Se debe propiciar, para poder continuar con esta propuesta, el que la educación en trabajo social provea para el desarrollo de destrezas en política social. En este sentido, con énfasis en la formulación y elaboración de política social como también en su implantación y evaluación. Es la noción general del proceso la que proveerá al estudiante del conocimiento, de la amplitud e importancia de esta dimensión necesaria a la educación en trabajo social.

Como los efectos de la globalización y la privatización con la cual lidia la sociedad en general atañen a todos, la educación en trabajo social debe enfatizar en su parcela de responsabilidad y en el compromiso de participación con las comunidades y con sus gentes, para responder a las exigencias y trastornos generados de estos fenómenos. En esta dirección no es suficiente desarrollar apresto, sino también destrezas para trabajar junto a las comunidades del país en la búsqueda de alternativas viables a la solución de sus problemas sociales, económicos y de otra índole, que consideran importantes.

\section{Propuestas para la Educación en Trabajo Social}

Durante los últimos años se han dejado sentir una serie de tendencias respecto a la educación en trabajo social que varían a lo largo de un continuo que va desde la educación tradicional, hasta la educación con un enfoque radical en sus propuestas para lidiar con la realidad.

Una de las propuestas más atractivas resurge nuevamente en los años 90. Ésta es la del Trabajo Social Radical, que tiene su inicio en los años 70. De acuerdo a De María (1992), el resurgimiento del enfoque radical se apoya grandemente en el nuevo resurgir del conservadorismo político. Comenta el referido autor que, de modo general, los educadores en trabajo social enseñan en escenarios 
institucionales amparados por el conservadorismo, el elitismo y el credencialismo. Que el trabajo es dirigido a un cuerpo de estudiantes, que junto a sus profesores son, con frecuencia, ideológicamente distantes de la gente, cuyas desventajas y circunstancias de vida, justifican el trabajo social en primer lugar.

Esta situación aludida por De María (1992) trae a su vez una serie de problemas en el enfoque curricular. Entre otros, señala los siguientes: a) ¿Cómo desarrollar y defender el trabajo social radical ubicado dentro de un currículo ortodoxo?, b) Los aspectos éticos a ser dilucidados entre educadores con un enfoque radical y los no radicales, y c) Los obstáculos de las agencias y del propio gobierno a las propuestas del trabajo radical.

La propia propuesta radical, según dicho autor, es difícil de ser introducida cuando el proceso propiciado por su desarrollo e implementación va contra la propuesta de opresión hegemónica de la sociedad. Comenta, "no se puede esperar llegar a la transcendencia radical, a no ser que se acepte el carácter dialéctico del proceso de educación".

A grandes rasgos, la propuesta de educación en trabajo social radical considera los siguientes puntos como neurálgicos:

1. El trabajo social es ambas cosas, radical y accionable. Es radical en el sentido de que va acompañado por una noción de ayuda transformadora y no reformadora.

Propone el cambio que busca alterar el carácter básico constitutivo de la sociedad. Considera en este sentido la dimensión ideológica, económica, de producción y de relaciones, que deben ser propiciadas y propulsadas desde el salón de clases.

2. El trabajo social debe desarrollar conocimientos y destrezas para servir a situaciones y gentes en contextos específicos. Al respecto, sugiere trabajar los cursos, de modo que éstos preparen al estudiantado en el enfoque radical paulatinamente a través del currículo.

3. El aprendizaje del estudiantado en trabajo social debe basarse en la contradicción, como por ejemplo, entre: 
- darle poder al cliente vs. la agencia que lo retira;

- propiciar auto determinación del cliente vs. el autoritarismo de las prácticas del bienestar social;

- la prestación de cuentas del cliente vs. la prestación de cuentas de la agencia;

- la teoría de trabajo social vs. la experiencia del estudiante;

- comenzar donde el cliente está vs. comenzar donde está el currículo de la Escuela;

- emitir juicios valorativos del cliente vs. exponerlos a evaluaciones de acuerdo a los criterios del sistema.

Se refiere aquí a la desmonopolización de los valores donde la propuesta radical, por no estar alineada ideológicamente, puede obtener su energía del socialismo, del humanismo o de la religión, etc.

4. La propuesta radical incide en un análisis radical que, para el autor, implica en acción. El análisis radical es conocimiento tanto crítico cuanto dialéctico. Parte importante de este análisis es la acción reflexiva. Ésta representa, de acuerdo a De María (1992), una gran correlación interdependiente entre lo que uno hace y lo que uno piensa acerca de lo que uno hace. Se requiere, en este sentido, de retroalimentación.

5. Recomienda el uso de historias polémicas para aclarar puntos de vista o situaciones y para trabajar la propia contradicción. Se recomienda este uso al máximo en el salón de clase y con relación a la propia instrucción práctica.

6. Sugiere centrarse en la marginalidad por considerarla de vital importancia al trabajo social, especialmente para ayudar a establecer límites de lo que es o no radical.

En otro de sus trabajos, el referido autor propone que maestros, estudiantes y clientes de trabajo social pueden y deben participar juntos en la construcción de marcos de referencia que reflejen y actúen críticamente sobre las construcciones dominantes de la sociedad.

Ciertamente la propuesta del trabajo social radical no deja de ser 
atractiva y ha sido parcialmente, y en casos de modo amplio, considerada en el desarrollo de currículos de escuelas de trabajo social en este hemisferio durante los últimos años. En cierta medida, esta propuesta concuerda con las propuestas del posmodernismo en el trabajo social. En este sentido se hace referencia a un trabajo recientemente presentado en la $40^{\text {ma. }}$ reunión del Consejo de Educación en Trabajo Social, llevado a cabo en Atlanta, Georgia, Estados Unidos. En este evento se reunieron educadores en trabajo social de toda esa nación y de otros países evaluados por este organismo privado, que se preocupa por la excelencia en la educación en trabajo social. Las propuestas allí presentadas variaron desde el servicio social clínico, al trabajo social que vende sus servicios privados, hasta la consideración de los posmodernistas. Estos últimos (Moore y Wallace, 1994) entienden y proponen que el trabajo social debe tener como meta la transformación de las estructuras de poder. Que la educación en trabajo social, para considerarse emancipadora, debe propiciar el desafío. En este sentido, entienden que la educación en trabajo social debe enseñar a la gente cómo retar a los opresores y a entender por qué son oprimidos. Se debe propiciar la amplia discusión de la opresión, incluso dentro del ciclo de vida familiar, de modo que la opresión no se vea como un fenómeno aislado y distante de las realidades de la cotidianidad. De la misma forma, esta propuesta rechazó enérgicamente la codificación de los comportamientos humanos por considerarlos reduccionistas. Argumentaban que todos los seres humanos actúan diferente y que se debe dar crédito a la variedad. Entre otras cosas, el grupo propuso extremo cuidado en la educación en trabajo social respecto a las políticas públicas, efectos de las cuales les provocan una mayor preocupación, pues de modo general tienden a ser, no sólo orientadas en términos político-partidistas, sino opresoras. Los posmodernistas, en su intento por ayudar al trabajo social a ser emancipador, hacen hincapié en la necesidad de enfatizar en el modelo de fortalezas en vez de en el modelo deficitario. Algunas son incluso hostiles contra minorías y grupos oprimidos por la sociedad. Al respecto, en el contexto latinoamericano, comenta Villa de Yarce (1992): "se requiere un rediseño de las políticas públicas para el sector social, rediseñar la participación de la población y de sus organizaciones en la implantación de programas". 
Invitaba la referida autora, de la misma forma, a ver la clientela como gente que puede reflexionar e influenciar nuestra práctica y que debían ser, de modo general, parte del proceso evaluativo. En esta línea de acción sugiere que la educación y práctica del trabajo social debería enfocar el trabajo con la clientela desde su propia realidad y contar con sus fortalezas.

En esta línea de pensamiento Rottier (1990), al referirse a la Formación Profesional del Trabajo Social en América Latina, dice y cito: "Requerimos actuar con una permanente reflexión crítica en oposición al activismo que acarrea la pérdida de perspectivas". Sugiere, también, incorporar la investigación como condición indispensable para el avance de la profesión. De la misma forma, sugiere esta autora

aprender el significado social de la profesión, comprendiendo claramente las determinaciones estructurales en las cuales debe situarse la explicación fundamental de los problemas sociales con los cuales los trabajadores sociales se vinculan cotidianamente.

Apoyando de la misma forma esta noción de educación en trabajo social, la educadora puertorriqueña, Dra. Raquel M. Seda Rodríguez, comentaba en su conferencia "Desarrollo Integral del Pueblo Puertorriqueño", en el Primer Congreso de Trabajo Social de Puerto Rico y el Caribe, 1987:

Hay que romper con la doctrina que adscribe virtualmente todos los problemas al cliente de los servicios, a deficiencias en el desarrollo de su personalidad y a relaciones familiares.

Argumentaba que esto era parte de una ideología política que no era otra cosa que un reduccionismo psicológico. Entendía que se patologizaba la pobreza y al oprimido, por ende a la desigualdad. Siendo el problema uno individual y no de la sociedad, justifica para el trabajo social campos de acción profesional individualizados e insuficientes.

De la misma forma, abogaba la doctora Seda Rodríguez (1984) que había que romper con el mito de que el trabajador social es un agente de cambio. En el manejo de casos los trabajadores sociales son facilitadores de cambio. Son las gentes con las que se trabaja, las que a nivel individual y colectivo tienen ese poder para transformar 
sus propias realidades existenciales. Comentaba, "nuestra labor es propiciar que se descubra ese poder y se utilice positivamente". Sugiere, al respecto, enmarcar la educación y la política de trabajo social en el contexto geográfico-histórico que la comprende.

Una serie de educadores y teóricos del trabajo social continúan, en los últimos años, proponiendo alternativas para la educación en trabajo social que ciertamente llaman la atención y merecen ser estudiadas. Gould (1996) propone una educación en trabajo social que se apoye en el paradigma del aprendizaje reflexivo. A su modo de entender, el aprendizaje reflexivo ofrece a la educación en trabajo social un acercamiento que opera a través del entendido de que el conocimiento profesional es inicialmente desarrollado a través de la práctica y del análisis sistematizado de la experiencia.

Las propuestas de Gould se apoyan en los trabajos de Donald Schön, a su vez influenciado por Dewey, quien identificó el foco de la reflexión como el proceso a través del cual toma lugar el aprendizaje por experiencia. Siendo así, la experiencia práctica es el foco organizador para el aprendizaje. De esta forma se refuerza la noción, ya tradicional en la educación en trabajo social, de que a través de la práctica es que el trabajador social genera su conocimiento y que lo validará a través de la sistematización de su propia práctica. De su trabajo se desprende que epistemológicamente existe equivalencia entre la práctica como generadora de conocimiento y la investigación científica, parámetro positivista para tales fines en las ciencias sociales.

Para Gould, el aprendizaje reflexivo reconoce que el propósito de la educación en trabajo social es facilitar el que las personas aprendan a desafiar el contexto normativo de la práctica, a sus pro-activos $\mathrm{y}$ adaptativos, dentro de un ambiente profesional en constante evolución. Debe aprender a ver la teoría como una reflexión, a su vez en proceso de transformación en la práctica.

Bismo y Cox (1997), preocupados con la tensión existente de modo tradicional entre el trabajo social y el ambiente, proponen que para poder responder adecuadamente a los requisitos del medio ambiente, la educación en trabajo social debe preparar profesionales capaces de lidiar efectivamente con lo que la sociedad y su gente necesita. Para tal propósito, proponen el desarrollo de cursos que 
promuevan el análisis crítico y la búsqueda de los principios e ideologías subyacentes; que aprendan a identificar principios sólidos del trabajo social en las áreas de conocimiento profesional entre la disciplina y la práctica; darle coherencia interna y homogeneidad a los cursos de práctica; y desarrollar cursos donde se apliquen los conceptos de las ciencias sociales a la práctica del trabajo social. Al respecto recomendaron los siguientes temas para ser considerados, entre otros: sociología del conocimiento, dinámicas del cambio social, teorías de estratificación social, mecanismos de control social, teorías de economía, políticas de tasación y sus efectos, análisis de presupuestos, ideología y políticas asistencialistas, análisis demográfico y políticas de población. Desde su perspectiva estos conocimientos podrían viabilizar una mejor y más efectiva intervención profesional, en el contexto actual cambiante donde se inserta el trabajador social.

Preocupados con las respuestas de la educación en trabajo social Medgey y Livermore (1997) sugieren el desarrollo de un modelo de desarrollo social. Este modelo apoyaría al trabajo social con su constante desafío por responder a los muchos cambios de las condiciones sociales existentes. Entienden que esta perspectiva de desarrollo social es una alternativa pro-activa y políticamente viable, al intentar asegurar que los programas sociales pueden contribuir positivamente al desarrollo económico. A su vez, el modelo propuesto, a su modo de entender, puede provocar intervenciones sociales que afecten positivamente la economía. Proveerá a los educadores en trabajo social la posibilidad de preparar estudiantes con capacidad de desarrollar acercamientos innovadores y pro-activos, que respondan a las realidades políticas y económicas de los tiempos actuales. Propone el desarrollo de concentraciones en desarrollo social y la interrelación de los conceptos aliados a este modelo, en los cursos de política social, organización de la comunidad y cursos clínicos también.

Esta propuesta es apoyada, dada su amplitud, por los trabajos de Berg-Weger y Schneider (1998), al proponer continuar con el desarrollo del trabajo interdisciplinario en trabajo social. Este tipo de trabajo tiene un gran potencial tendiente no sólo a la colaboración, como también al fortalecimiento en la identidad profesional del trabajador social. A su vez, apoya el empoderamiento de los educadores y practicantes del trabajo social, hacia una más efectiva fusión. Entienden 
los autores que la colaboración interdisciplinaria en la educación en trabajo social - que se da más a nivel de estudios graduados- puede fortalecer el papel del trabajador social, tanto en lo académico como en la práctica.

Estudios llevados a cabo por estos autores apuntan a que según los recursos de la sociedad continúan disminuyendo para el ofrecimiento de los servicios sociales, el desarrollo de destrezas de colaboración efectiva se convierte en un asunto crítico de subsistencia para la profesión y los profesionales del Trabajo Social.

Esta moción de trabajo colaborativo es a su vez desarrollada por Bailey y McNally Koney (1996), a través de una propuesta de desarrollo de colaboración inter-organizacional de base comunitaria. Entendieron éstos que este acercamiento podría mejorar los vínculos entre agencias, consumidores de servicios sociales y muchas de las comunidades, para ampliar sus voces y su participación en la toma de decisiones en programas y desarrollo de políticas sociales.

Esta propuesta apunta a su vez hacia la necesidad de que la educación en trabajo social y la práctica comiencen a focalizar en colaboración. Abogan los autores por un acercamiento académico defensor de la práctica social que provea a los profesionales de destrezas y conocimientos relacionados a la investigación participativa para involucrar a las comunidades en actividades dirigidas al cambio social. Abogan por el desarrollo de formas que propicien la integración del aprendizaje obtenido en las comunidades a la academia, dándole así un acercamiento holístico a la práctica del trabajo social.

Todas estas propuestas y sus consiguientes requisitos en el contexto de la educación en trabajo social requieren de competencia profesional tanto en la enseñanza como en la práctica. Para O'Hagan (1996) esta competencia está insertada en el conocimiento, los valores y las destrezas propias del trabajador social. Desde su perspectiva, los trabajadores sociales deben ser capaces de conceptualizar, reflexionar, analizar y evaluar críticamente los cimientos básicos del conocimiento y de la práctica del Trabajador Social. Deben ser pensadores estratégicos dispuestos a pensar en las ventajas y desventajas de las propuestas y a poder anticipar las posibles consecuencias de sus decisiones y acciones.

Sin pensar de modo contrario, Popell (1996) en su trabajo, 
Conocimiento Teórico y la Naturaleza del Mundo Real, sugiere que el trabajador social debe usar su sabiduría profesional. Implicaba que el trabajador social necesita usar su propio juicio profesional, cuando el conocimiento no está disponible. Implica también que se le debe enseñar a los estudiantes a estar a tono y en sintonía con la realidad. Esto implica saber que los clientes están siempre moviéndose a lo desconocido y no es función de los trabajadores sociales determinar su destino. Es por eso que el juzgar, la intuición, el confiar en los instintos y la sabiduría profesional se dignifican y profundizan. Trabajar con fortalezas y respetar las decisiones humanas y la fragilidad, es más aceptable, y así se evita la patologización de clientes y poblaciones. En este sentido, la práctica profesional provee un apoyo concreto que eventualmente deberá ser validado por la sistematización de la propia práctica.

Preocupados con esta temática de la educación en trabajo social hubo una reunión de directores y decanos de trabajo social cuyo producto fue publicado por Mandel School of Applied Social Sciences (1996). Entre las preocupaciones y temas recurrentes de este evento, vale la pena mencionar las siguientes:

1. Desarrollar nuevos modelos en la teoría y la práctica que puedan operacionalizar de modo más efectivo la perspectiva de trabajo social que lidia con la persona en el ambiente social.

2. Llevar a cabo investigaciones de corte interdisciplinario que faciliten el desarrollo de nuevos modelos y el acercamiento a asuntos sociales controversiales.

3. Desarrollar esfuerzos de colaboración intra y extra universitarios en el campo de investigación social para investigar en qué forma se pueden combinar esfuerzos para acercarse a problemas semejantes y contrarrestar la indiferencia congresional y hasta su hostilidad, para monitorear el impacto de los cambios en la implantación de las nuevas políticas sociales y maximinizar el potencial de los limitados recursos disponibles.

4. Revivir la tradición de defensa de la profesión y continuar trabajando en pro de la justicia social y económica.

5. Re-examinar el valor de la tecnología en nuestra profesión, 
tanto en el campo de la educación como en el de la investigación en trabajo social.

Si se resumiese la propuesta en la educación en trabajo social ante los retos del Siglo XXI diríamos que: la educación en trabajo social debe ser una caracterizada por la excelencia educativa a nivel tanto teórico como práctico, que garantice un conocimiento actualizado y una ejecutoria de reconocida calidad. Debe estar esta educación, como se comenta anteriormente, enmarcada en el contexto de la realidad social nacional en la cual se desarrolla, para responder a sus necesidades, retos y desafíos. Debe, en esa dirección, apoyarse en lo que autores como De María, Seda Rodríguez, Rottier y otros apuntan, responder a las necesidades de la sociedad y de sus gentes.

Sobre todo debe ser una educación que responda de forma actualizada a su época y que esté comprometida con los retos que su propia circunstancia histórico-académica le exige. Debe también intentar anticiparse a las necesidades emergentes del pueblo, que es lo que Faherty (1997) aboga cuando dice que se hace necesario recurrir a métodos de predicción adecuados para adelantarnos a las necesidades académicas. Para esto debe basar el diseño de su currículo en una consulta amplia de la base a la cual sirve. Debe responder a desafíos como el HIV-SIDA y su impacto en la familia y la sociedad, la globalización, las drogas, la opresión y marginalidad, las cambiantes familias, en especial el creciente aumento en jefaturas femeninas, la criminalización del comportamiento, la militarización de lo cotidiano, los niños con llave, los niños de la calle, la pobreza, el cambio en los valores mesocráticos de la sociedad y la privatización de los servicios públicos, entre otros. Se requiere flexibilidad, adaptación y mucha creatividad, sin obviar, por supuesto, el presupuesto necesario para tales fines.

Metodológicamente, la educación en trabajo social debe continuar no sólo con los métodos tradicionales debidamente actualizados, sino también abrirse hacia nuevas dimensiones de acción como respuesta a requisitos propios de la comunidad a la que sirve. Específicamente se hace referencia al profesional de trabajo social que ofrece sus servicios como profesional libre y cobra por los mismos, al trabajador social que se incorpora a través de su propia 
empresa y vende servicios al Estado, a la comunidad, a los individuos y a otras empresas privadas. En este sentido se amplían grandemente las dimensiones del trabajo ofrecido, y las metodologías de la misma forma.

Se exhorta enfáticamente a enmarcar el trabajo social en marcos de referencia, teorías y modelos que le den apoyo, lo fortalezcan y, ¿por qué no?, que lo desafíen y que a la vez lo ayuden a sustentar su trabajo práctico de forma actuante y dinámica, en fin, comprometida.

Este nuevo impulso que toma la profesión, y por ende la educación, trae a colación un asunto considerado controversial en trabajo social. El mismo se refiere a si la misión de las escuelas, como la de la profesión, debe o no, estar orientada hacia el ofrecimiento de servicios privados. De todas formas la educación en trabajo social tiene ante sí estos y muchos otros retos que la obligan a ser reflexiva, flexible y receptiva ante la creciente complejidad y sus demandas.

Sobre todo la educación en trabajo social debe darle énfasis, especial a la preparación para la práctica profesional que evidencie excelencia. Esto requiere que la instrucción práctica sea colocada al mismo nivel que la educación teórica, en el pedestal que le corresponde, ofreciendo a los profesores de instrucción práctica igualdad de status, reconocimiento y paga. La práctica nutre la profesión y sin ella deja de ser y se anquilosa. Este punto debe ser repensado y colocado en la justa perspectiva, más ahora que se tiene tanta multiplicidad de situaciones y escenarios en el horizonte, tanto en el contexto público como en el privado. Responder a estos retos educativos es un gran desafío.

La complementación de esta situación educacional requiere que se considere como obligatoria la educación continuada formal para los educadores en trabajo social. Se recomienda también que los profesores se nutran de la práctica continuamente. A su vez, podrían elaborarse convenios donde se intercambiasen conocimientos teóricos con el peritaje práctico del personal de las agencias. Ciertamente este personal estará ávido de compartir sus experiencias prácticas apoyando así la academia, de la misma forma que la academia podría compartir su bagaje teórico. Se recomienda complementar la educación con ese componente práctico que nos es tan precioso. 


\section{Hacia dónde: Tendencias a ser consideradas en el desarrollo de currículos en Trabajo Social y otras áreas}

Conocido por todos es que la excelencia de los currículos estriba en su flexibilidad para responder a los requerimientos de los tiempos donde se insertan, se desarrollan, implementan e intentan generar un producto de impacto (Ríos de Caraballo, 1985).

Los tiempos actuales donde se es partícipe directo e indirecto de la globalización y de la privatización a nivel nacional e internacional, como también de sus efectos en la regionalización, ciertamente tendrán, como comenta Aponte (1997), impacto relevante en el rol de las instituciones de educación superior. Se vislumbra, de acuerdo al análisis del referido autor, una serie de tendencias que éste recoge en las tablas presentadas a continuación y que definitivamente tendrán su impacto en los currículos y todo lo que a ellos se refiera: La Tabla \#1, adaptada del trabajo de Aponte (1997), evidencia las tendencias generales sobre cambios organizacionales y curriculares hacia la postindustrialidad, a saber:

\section{Tabla \#1}

Tendencias generales de cambios organizacionales y curriculares con relación a las metas y objetivos de la educación superior de la postindustrialidad - sectores dinámicos de la economía

Hacia la postindustrialidad

- Integración de las disciplinas/subespecialidades - interdisciplinariedad

- Programa de estudios interdisciplinarios con flexibilidad curricular

- Educación continua conducente a grados y multiespecialidades

- Investigación práctica aplicada relacionada con el conocimiento aplicado a la producción de bienes y servicios

- Investigación interdisciplinaria utilizando varios lenguajes, códigos y variedad de sistemas de comunicación

- Destrezas simbólicas de conceptualización, análisis cuantitativo y visual relacionadas con el proceso de investigar, resolver problemas utilizando información y conocimiento

- Crecimiento de unos programas a expensas de otros e integración de programas; prioridad al aumento de programas científicos-tecnológicos 
- Evaluación y avaluación de los programas académicos a través de criterios de pertinencia y calidad de los nuevos requerimientos de la postindustrialidad con relación a la misión de las instituciones.

Las tendencias aludidas traen a colación una serie de dimensiones a las cuales hay que prestarle atención en el contexto de la educación en Trabajo Social. Ciertamente, su impacto se percibirá en modificaciones adaptativas que, sin ser extrañas, posiblemente reestructuren las orientaciones curriculares existentes agilizándolas para responder a las nuevas exigencias de estos tiempos cambiantes.

La siguiente Tabla \#2, relativa a cambios curriculares, de la misma forma, incidirá en cambios a varios niveles relacionados con la educación en Trabajo Social.

\section{Tabla \#2}

Tendencias de cambios curriculares, organizacionales y el nuevo rol de los docentes en la educación superior en la transición hacia la postindustrialidad

\section{Hacia la postindustrialidad}

- Docencia interdisciplinaria-colaborativa de equipos

- Docencia orientada hacia facilitar el proceso de aprendizaje en y fuera del aula

- Docencia/aprendizaje en la acción participativa, apoyada por tecnología y las comunicaciones; entre facultades, con otras instituciones (empresas, comunidad, gobierno, escuelas, el hogar, etc.) a distancia e interactiva

- Docencia investigativa apoyada por tecnología y las comunicaciones con fines prácticos orientada a la solución de problemas de actualidad

- Investigación y creación de conocimiento en la acción

- Flexibilidad de programación del período lectivo; integración y aplicación del conocimiento multidisciplinario en el currículo

- Docencia-gerencia y liderato institucional/comunitario

- Intelectualidad simbólica/pública internacional

Se observa concretamente el impacto de las tendencias en el tipo de aprendizaje, la investigación y las exigencias de flexibilidad programática. Un alerta que la profesión y, principalmente, los 
educadores no deben ignorar, pues implica en re-enfoques en la docencia y metodologías de trabajo, a los cuales se debe reaccionar como profesión y como profesionales de la educación en trabajo social.

En una línea de pensamiento semejante a ésta, Barrow (1997) dice que los crecientes cambios económicos —una de las variables intervinientes de las que se ha hablado-están llevando a los líderes y administradores universitarios, entre otras cosas, a transformar sus currículos y a darle una nueva dirección a los limitados recursos disponibles. Entre los temas aludidos está el relativo al impacto de la transformación del trabajo y sus repercusiones, hacia que los currículos se modifiquen y amplíen sobre el desarrollo de destrezas interdisciplinarias.

A su modo de entender, la educación superior debe prepararse para un producto que sea capaz de resolver problemas, que sea flexible y con una gran capacidad de análisis, y sobre todo, enfatizando la destreza en la solución de problemas. Además, sugiere que este producto sea uno capaz de combinar destrezas de pensamiento crítico con conocimientos especializados, y poder aplicarlos.

Lo antes expuesto resulta interesante en la medida en que algunas de estas condiciones convergen entre campos profesionales diferentes, como son la economía y el trabajo social, que a pesar de éstas, se complementan en la realidad práctica del cotidiano. En esta línea de pensamiento, el autor propone que los currículos se desarrollen hacia matrices de destrezas. Se refirió concretamente a varias que parecieron pertinentes al trabajo social, por lo menos para analizarlas en sus méritos, y son las siguientes:

- desarrollar currículos que doten al individuo de destrezas flexibles asociadas a identificar y solucionar problemas, entre otras,

- desarrollar y apoyar el enfoque interdisciplinario

- propiciar el desarrollo de currículos integrados, coherentes y conectados, que permitan transferir destrezas y conocimientos de una área a otra.

En el campo concreto de Trabajo Social, Jarman-Rhodes y otros (1997) alertan sobre los posibles efectos de medidas propuestas por el gobierno en su afán por balancear los presupuestos, cortar los 
el gobierno en su afán por balancear los presupuestos, cortar los gastos de salud y distribuir los fondos federales a los estados en bloque. Sugieren los autores considerar todo esto, además de los impactos que la globalización traerá; a su modo de entender, cambios en la naturaleza y organización de los servicios humanos. Dada la condición político-económica de Puerto Rico esta propuesta afectará en gran medida todas las dimensiones de la sociedad.

Alertan, también, sobre otra serie de aspectos que afectan la práctica y a su vez la educación en Trabajo Social como lo son: la reducción de costos, las iniciativas de cuidado dirigido y la reorganización de agencias. El cuidado dirigido, a su modo de entender, ha llevado al rediseño de servicios rápidos y orientados hacia el lucro. Ciertamente, variables intervenientes, sobre las cuales se debe reaccionar desde la perspectiva de la profesión de Trabajo Social.

\section{Referencias}

Aponte, E. (1997). Educación Superior, Trabajo y la integración económica del Merconorte; Escenarios tendenciales de cambio para las instituciones de la Región de las Américas, el Caribe y Puerto Rico. CHE's News, 1(3), 6-9.

Barley, D. y McNally-Koney, K. (1996). Inter-organizational Community-Based Collaboration. A Strategic Response to Shape the Social Work Agenda. Journal of the National Association of Social Workers, 41(6), 602-612.

Berg-Weger, M. y Schneider, F.D. (1998). Interdisciplinary Collaboration in Social Work Education. Journal of Social Work Education, 30(1), 97-107.

Bernabe Riefkohl, A. (1994). Back to the Future Globalization and Legal Education. Trabajo mimeografiado sin publicar.

Bismo, H. y Cox, F. (1997). Social Work Education: Catching up with the present and the future. Journal of Social Work Education, 33(2), 373-387.

Barrow, C.W. (1997). The New Economy and the New Curriculum Trends in U.S. Higher Education, CHE's News, 1(3), 3-4.

De María W. (1992). On the Trial of a Radical Pedagogy for Social Work Education, British Journal of Social Work, 22, 231-252.

Faherty, V.E. (1997). Using Forecasting Models to Plan for Social Work Education in the Next Century. Journal of Social Work Education, 33(2), 403-411.

Gould, N. (1996). Social Work Competence: On Historical Perspective in K. O'Hagan, 
Ed. Competence in Social Work Practice. London Jessica Kingsley Publishers.

Guardiola Ortiz, D. (1993). Comentarios en torno a la ponencia de la licenciada

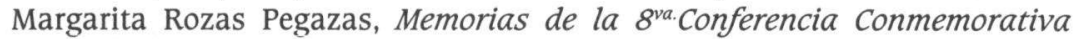
Dorothy D. Bourne. Río Piedras, Escuela Graduada de Trabajo Social.

Jarman-Rhodes, L. y otros (1997). The Changing Context of Social Work Practice: Implications \& Reccomendations for Social Work Educators: Journal of Social Work, 3(1): 29-46.

Mandel School of Applied Social Sciences (1996). White Paper on Social Work Education. Today and Tomorrow. Ohio Mandel School of Applied Social Sciences.

Migdley, J. y Livermore, M. (1997). The Developmental Perspective in Social Work, Educational Implications for the Next Century, Journal of Social Work Education, $33(3), 573-585$.

Molina Molina, L. y Guzmán Stein, L. (1992). Perspectivas del trabajo social costarricense para el año 2000 y características del perfil profesional: Acción Crítica, 27: 21-63.

Moore, L.S. y Wallace, G. (1994). Emancipatory Social Work: The Implications of PostModern Theory to Social Work. Trabajo presentado en la 40 ${ }^{\text {ma. }}$ Reunión Anual del Council on Social Work Education, Atlanta, Georgia.

Morales, A.T. y Sheaford, W.B., Eds. (1995). Social Work- A Profession of Many Faces. Boston: Allyn \& Bacon.

O'Hagan, K. (1996). Social Work Competence: An Historical Perspective: K. O'Hagan, Ed., Competence in Social Work Practice. London Jessica Kingsley Publishers.

Papell, C.P. (1996). Reflections on Issues of Social Work Education: N. Gould, e I. Taylor (Eds.) Reflective Learning for Social Work. England: Arena.

Ríos de Caraballo, J.R., (1995). La Excelencia en la Educación en Trabajo Social: Un reto para la participación estudiantil. Ponencia inédita. Río Piedras: Escuela Graduada de Trabajo Social.

Rottier, N.H. (1990). Celebremos el XXV Aniversario del ALAETS y el XV Aniversario de CELATS: Acción Crítica, 27: 5-9.

Rozas Pegazas, M. (1993). Crisis Económica, Estado y Política Social: Su impacto en el sistema de Bienestar Social: Memorias de $8^{\text {va. }}$ Conferencia Conmemorativa Dorothy D. Bourne. Río Piedras, Escuela Graduada de Trabajo Social.

Seda Rodríguez, R.M. (1987). El desarrollo integral del pueblo puertorriqueño: Reto del Trabajador Social. Trabajo presentado en Ponencia Congreso de Trabajo Social de Puerto Rico y el Caribe, San Juan, Puerto Rico.

Strom-Gothfied, K. (1997). The Implications of Managed Care for Social Work Education. Journal of Social Work Education, 33(2), 403-411.

Villa de Yarce, L.M. (1992). La responsabilidad del Trabajo Social en la construcción de las sociedades democráticas y modernas de América Latina: Acción Crítica, 31:59-74. 
